\title{
Local staging of rectal cancer using fused high resolution diffusion weighted imaging and modified MR rectography
}

\author{
Xue Tang ${ }^{1}$, Yan Luo ${ }^{1}$, Shipai Zhang ${ }^{2}$, Ligang Xia ${ }^{2}$, Jingshan Gong ${ }^{1}$ \\ ${ }^{1}$ Department of Radiology, ${ }^{2}$ Department of Gastrointestinal Surgery, Shenzhen People's Hospital, the Second Clinical Medical College of Jinan \\ University, Shenzhen 518020, China
}

Correspondence to: Jingshan Gong. Department of Radiology, Shenzhen People's Hospital, the Second Clinical Medical College of Jinan University, Shenzhen 518020, China. Email: jshgong@sina.com.

\begin{abstract}
Rectal cancer (RC) is a common malignant tumor with high mortality. MR imaging plays an important role in treatment decision making of RC. Unfortunately, the contents (gas and feces) in the rectum often induce artifacts and thus negatively affect the depicting and staging of RC. We developed a new protocol for MR rectography using oral administration of iso-osmotic mannitol to distend lumen after bowel cleansing preparation. Fused MR rectography and high resolution diffusion weighted imaging (DWI) is then performed to facilitate detection and staging of RC. Our present technique can eliminate the effect of gas and feces on image quality, especially on DWI, and can achieve satisfactory bowel distention, lesion depicture and visualization of surgical planes. Fused high resolution DWI and MR rectography can be a promising technique to improve the accuracy of RC local staging.
\end{abstract}

Keywords: Rectal cancer (RC); MR imaging; rectography; high resolution diffusion weighted imaging (DWI); fusion

Submitted Jun 28, 2019. Accepted for publication Jul 29, 2019.

doi: 10.21037/qims.2019.08.02

View this article at: http://dx.doi.org/10.21037/qims.2019.08.02

\section{Introduction}

Rectal cancer (RC) is a common cancer with high mortality $(1,2)$. Local staging of RC, including the status of circumferential resection margin (CRM), depth of tumor spread and adjacent lymph node involvement, is one of the factors influencing the patients' survival (2). Accurate staging using advanced imaging technique can facilitate clinicians for the patients' treatment decision making and improve the patients' outcomes (3-6). MR imaging has been proved to be the first-line imaging modality to stage and assess the response of neoadjuvant radiotherapy and chemotherapy (6). Unfortunately, rectum is a luminal organ, which is often filled with gas and feces or is at the status of collapse. The gas and feces can not only influence the visualization of the lesions, but also introduce susceptibility artifact due to gas-tissue interface effect. Furthermore, collapse of lumen may prevent demarcating the lesion from normal tissue clearly. MR colonography or rectography were developed using bright-lumen (a gadolinium chelatespiked enema) or dark-lumen (gas) techniques to distend colorectum $(7,8)$. Both techniques have some limitations. Bright-lumen technique may influence assessing tumor enhancement, and the dark-lumen technique may introduce susceptibility artifacts. Ultrasonographic gel has been introduced to distend colorectum lumen and proved to be an effective method to facilitate lesion depiction within the wall and its extension estimation (9-12). The injection pressure can result in over distention of rectum, which may alter the distance between the tumor and surgical planes (13). This distance has a crucial role on assessing the involvement of the mesorectal fascia (MRF) by tumors. The distance of less than $1 \mathrm{~mm}$ is regarded as an involvement of MRF, and not suitable for total mesorectal excision. The introduction of ultrasonographic gel needs to insert a rectal tube on the MR examination bed. Therefore, it will increase patients' 


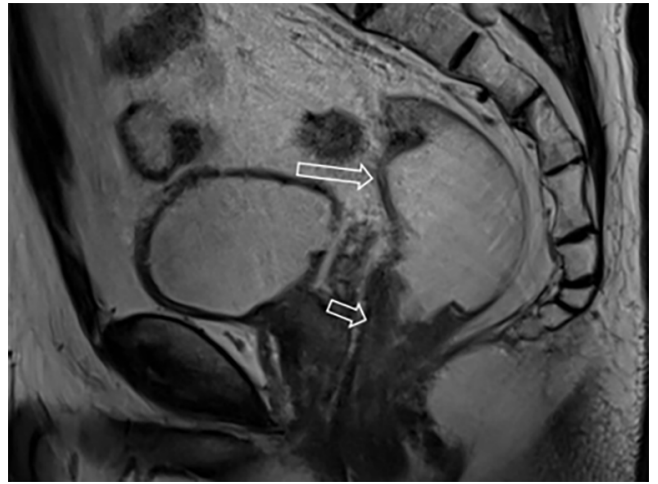

Figure 1 A 64-year-old man with low RC. Sagittal MR rectography shows satisfactory distention of the upstream rectal lumen (long arrow) and the tumor (short arrow). RC, rectal cancer.

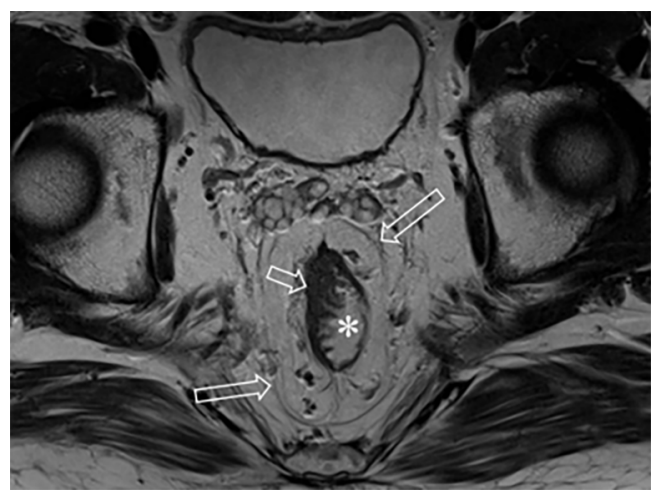

Figure 2 A 67-year-old man with low RC. Axial MR rectography shows excellent distention of rectal lumen (asterisk), clear mesenteric fascia (long arrows) and the stage T2 tumor (short arrow). RC, rectal cancer.

examination room time, and the effect of feces can't be eradicated completely. We developed a novel method to perform MR rectography through oral administration of isosmotic mannitol after bowel cleansing preparation; and fusion of high resolution diffusion weighted imaging (DWI) and MR rectography was carried out to improve local staging of RC.

\section{Technique and protocol of MR rectography}

This study was approved by institutional review board and obtained informed consent from all the patients. Patients undergoing MR rectography were asked to take compound polyethylene glycol electrolyte orally as laxative on the night prior to the exam to clear the colorectum. The examinations were scheduled in the morning. About $1,500 \mathrm{~mL} 52$ of isosmotic mannitol was continuously taken orally in 53 90 to 120 min before examination. MR rectography was 5 performed when the patients had the feeling of bowel 55 movement again after 2 or 3 excretions. To ensure the 56 completeness of the examination, the patients were permitted to have two or three bowel movements before the beginning of examination. All the preparation was performed outside the examination room. Therefore, it did not increase examination time and did not influence the patient flow. MR rectography was performed at rectal axial, coronal and sagittal high resolution T2 weighted imaging at 3.0T MR system (Magnetom Skyra, Siemens Healthcare, Erlangen, Germany) using parameters as follows: repetition time (TR), 3,000-4,200 ms; echo time (TE), 83-101 ms; slice thickness, $3 \mathrm{~mm}$; field-of-view, 220-280 mm; matrix, 381-435 $\times 448-512$. Besides the regular dynamic contrast enhanced MR imaging, high resolution DWI was obtained using the techniques of readout segmentation of long variable echo trains (RESOLVE) at transverse and sagittal planes with the following parameters: TR, 5,800-7,030 ms; TE,61 ms; slice thickness, $3 \mathrm{~mm}$; field-of-view, $220-230 \mathrm{~mm}$; matrix, 116×116; and b values, 0 and $1,000 \mathrm{~s} / \mathrm{mm}^{2}$. Fusion of high b value DWI onto T2WI was conducted by using an image processing workstation (Syngo Via, Siemens Healthcare, Erlangen, Germany) at both axial and sagittal planes respectively.

\section{Results and discussion}

After the cleansing preparation, effect of feces on the lesion depicture of CRC can be eradicated completely. Intention of bowel movement shows that the contrast material has reached the rectum. Therefore, luminal distention can be obtained also, which can improve tumor visualization (Figure 1). With high resolution T2 weighted images on axial, coronal, and sagittal planes, this technique also showed its advantage in displaying the relationship of tumor to surgical planes, such as CRM and anal sphincter (Figures 2-4).

DWI has been proved to a valuable tool for tumor detection and characterization staging, prognosis evaluation, assessing response to treatment and recurrence of RC. Due to high cellularity and heterogeneity, RC appears as high signal lesion on high b value DWI. Unfortunately, gas in non-preparation bowel can induce susceptibility artifact to distort the images (Figure 5). In present protocol, oral isosmotic mannitol after bowel cleansing preparation can

54 57 53 55 56 


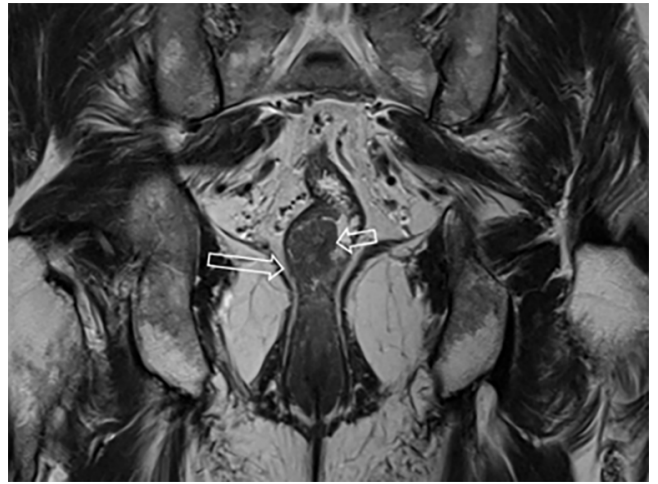

Figure 3 A 59-year-old man with low RC. Coronal MR rectography shows the tumor (short arrow) and clear anal sphincter space (long arrow). RC, rectal cancer.

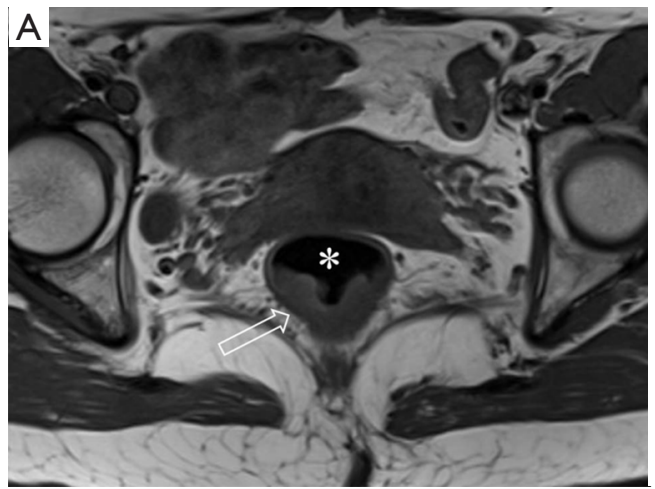

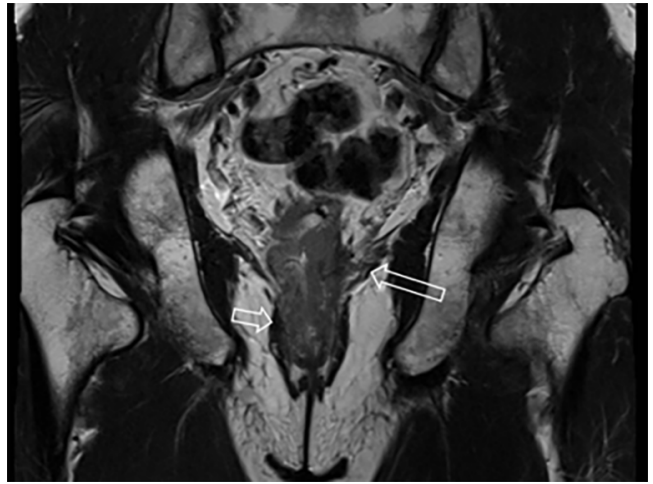

Figure 4 A 60-year-old man with low RC. Coronal MR rectography shows that external anal sphincter (short arrow) and levator ani muscle (long arrow) are invaded. RC, rectal cancer.

Figure 5 A 51-year-old woman with low RC. (A) Axial MR rectography shows the tumor (short arrow) and the air in the rectum (asterisk) at the T2WI; (B) axial DWI shows the tumor (arrow) is distorted due to air-tissue interface susceptibility artifact. RC, rectal cancer; DWI, diffusion weighted imaging.
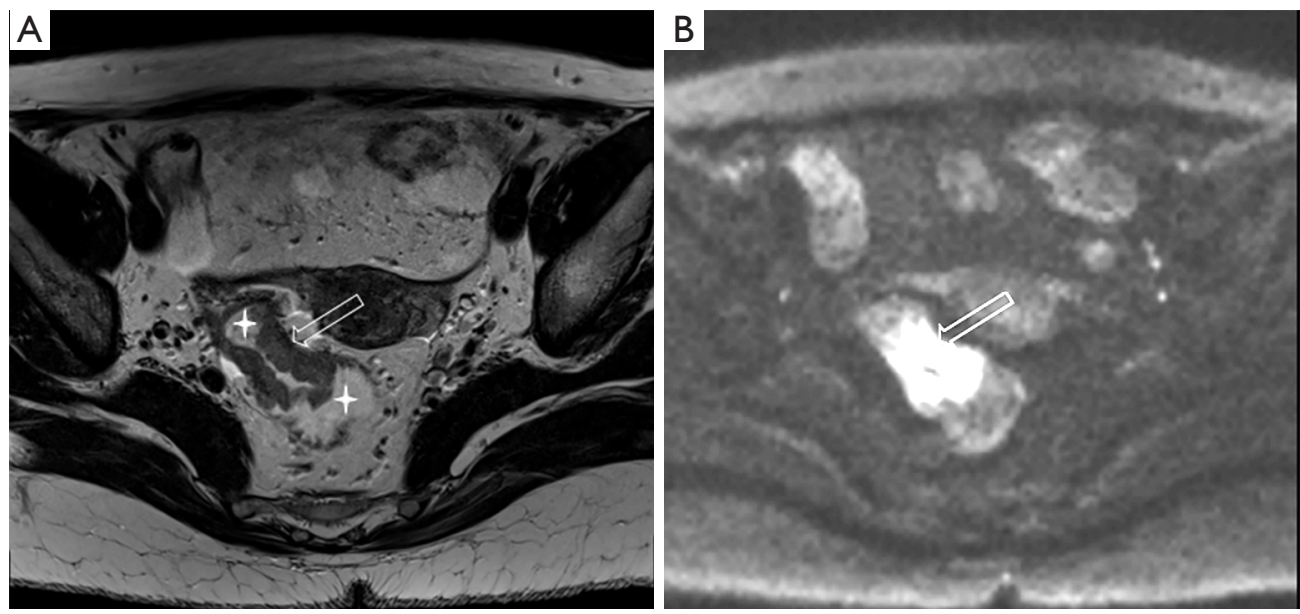

Figure 6 A 54-year-old man with high RC. (A) Axial MR rectography shows the tumor (arrow) and the well filled rectum (asterisk); (B) axial DWI demonstrates the tumor (arrow) with clarity. RC, rectal cancer; DWI, diffusion weighted imaging. 


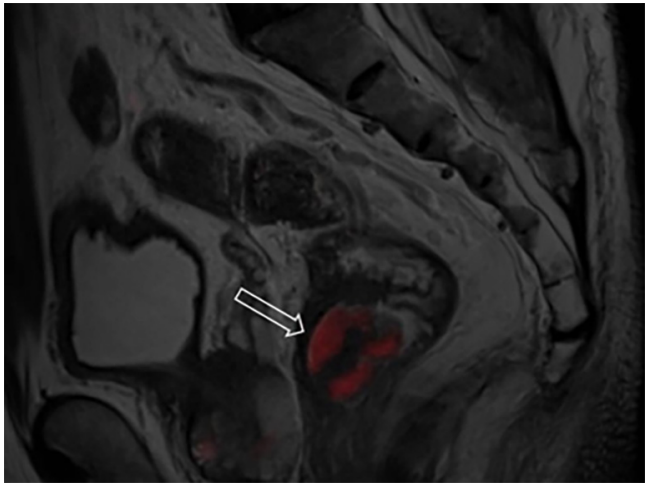

Figure 7 A 60-year-old man with low RC. Fused sagittal MR rectography and DWI shows the tumor with restricted diffusion in red color (arrow) without breaking through the outer membrane of the rectum. RC, rectal cancer; DWI, diffusion weighted imaging.

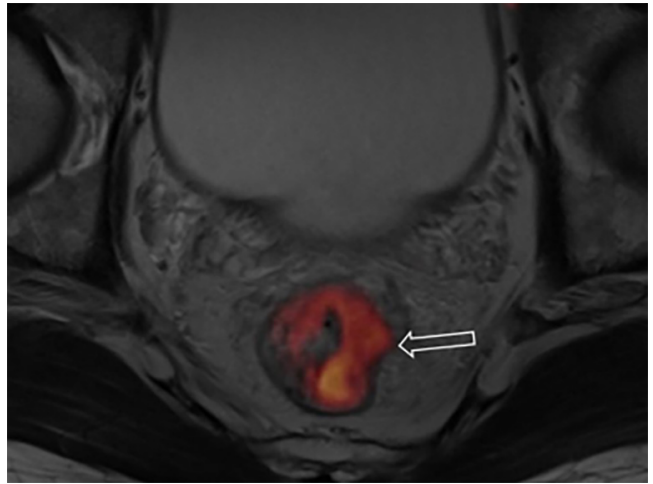

Figure 8 A 55-year-old man with low RC. Fused axial MR rectography and DWI shows a stage T3a tumor (arrow) in hot color which has broken through the outer membrane of the rectum. RC, rectal cancer; DWI, diffusion weighted imaging.
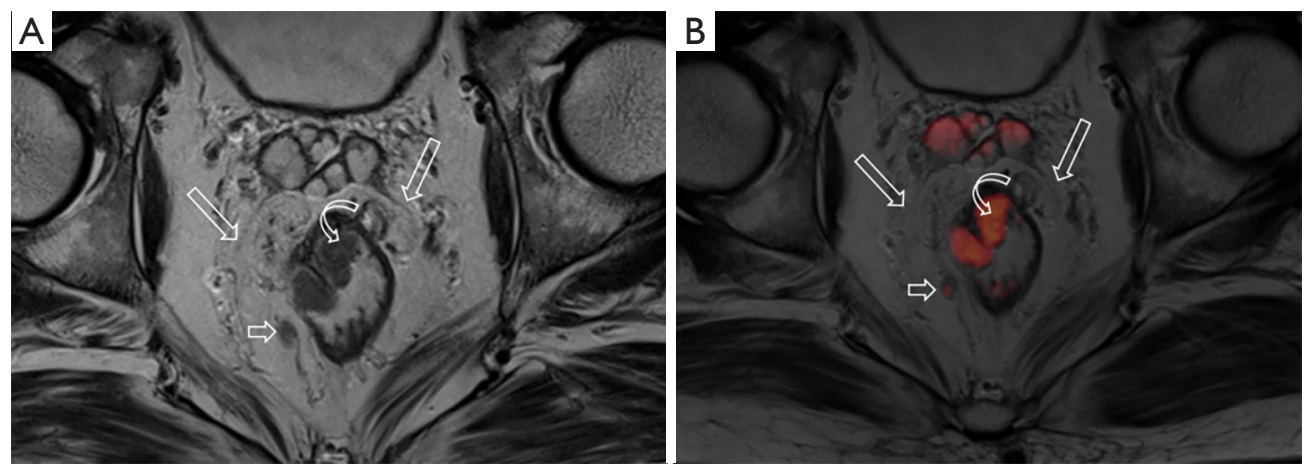

Figure 9 A 59-year-old man with low RC. (A) Axial MR rectography shows the tumor (curve arrow), enlarged lymph nodes (short arrows) and clear mesenteric fascia (long arrows); (B) fused axial MR rectography and DWI visualizes the tumor (curve arrow) and enlarged lymph nodes (short arrows) with restricted diffusion in color. The clear mesenteric fascia (long arrows) can be also identified. RC, rectal cancer; DWI, diffusion weighted imaging.

eliminate gas inducing artifact on DWI (Figure 6). To overcome lower spatial resolution, RESOLVE technique and fusion of DWI and MR rectography were used to improve spatial resolution of DWI and to combine advantages of DWI in visualization of lesions and high spatial resolution of T2WI (Figure 7). This technique could not only improve the assessment of the tumor's position and the local invasion (Figure 8), but also facilitate evaluation of adjacent lymph nodes, for metastatic nodes of which often exhibit significant diffusion restriction (Figure 9).

In conclusion, we described a new protocol for MR rectography, which uses isosmotic mannitol instead of gas to distend the bowel lumen after bowel cleansing. This was shown to be a practical method to improve image quality and lesion depicture of RC through eliminating effect of gas and feces. Another advantage is that our method can eliminate susceptibility artifact induced by gas interface so that it is very suitable for diffusion weighted sequence. Fusion of high resolution DWI and MR rectography, which combines the advantages of these two sequences, is a promising technique to improve the accuracy of RC local staging.

\section{Acknowledgments}

Funding: This work was supported by Shenzhen Science 
and Technology Program (No. JCYJ20180301170121400).

\section{Footnote}

Conflicts of Interest: The authors have no conflicts of interest to declare.

\section{References}

1. Siegel RL, Miller KD, Jemal A. Cancer Statistics, 2017. CA Cancer J Clin 2017;67:7-30.

2. Siegel RL, Miller KD, Fedewa SA, Ahnen DJ, Meester RGS, Barzi A, Jemal A. Colorectal cancer statistics, 2017. CA Cancer J Clin 2017;67:177-93.

3. Feinstein AR, Sosin DM, Wells CK. The Will Rogers phenomenon. Stage migration and new diagnostic techniques as a source of misleading statistics for survival in cancer. N Engl J Med 1985;312:1604-8.

4. Hillner BE, Siegel BA, Liu D, Shields AF, Gareen IF, Hanna L, Stine SH, Coleman RE. Impact of positron emission tomography/computed tomography and positron emission tomography (PET) alone on expected management of patients with cancer: initial results from the National Oncologic PET Registry. J Clin Oncol 2008;26:2155-61.

5. Shahrier M, Ahnen DJ. Colorectal cancer survival in Europe: the Will Rogers phenomenon revisited. Gut 2000;47:463-4.

6. García-Figueiras R, Baleato-González S, Padhani AR, Luna-Alcalá A, Marhuenda A, Vilanova JC, OsorioVázquez I, Martínez-de-Alegría A, Gómez-Caamaño A. Advanced Imaging Techniques in Evaluation of Colorectal Cancer. Radiographics 2018;38:740-65.

Cite this article as: Tang X, Luo Y, Zhang S, Xia L, Gong $\mathrm{J}$. Local staging of rectal cancer using fused high resolution diffusion weighted imaging and modified MR rectography. Quant Imaging Med Surg 2019;9(9):1592-1596. doi: 10.21037/ qims.2019.08.02
7. Thornton E, Morrin MM, Yee J. Current status of MR 156 colonography. Radiographics 2010;30:201-18. 157

8. Agildere AM, Tarhan NC, Ergeneli MH, Yologlu Z, 158 Kurt A, Akgun S, Kayahan EM. MR rectography 159 evaluation of rectoceles with oral gadopentetate $\quad 160$ dimeglumine and polyethylene glycol solution. Abdom $\quad 161$ Imaging 2003;28:28-35.

9. Macario S, Chassang M, Novellas S, Baudin G, Delotte 163 J, Toullalan O, Chevallier P. The value of pelvic MRI 164 in the diagnosis of posterior cul-de-sac obliteration in $\quad 165$ cases of deep pelvic endometriosis. AJR Am J Roentgenol 166 2012;199:1410-5.

10. Palmucci S, Piccoli M, Piana S, Foti PV, Siverino ROA, 168 Mauro LA, Milone P, Ettorre GC. Diffusion MRI for $\quad 169$ rectal cancer staging: ADC measurements before and $\quad 170$ after ultrasonographic gel lumen distension. Eur J Radiol 171 2017;86:119-26.

11. Lambregts DM, Beets GL, Maas M, Curvo-Semedo L, 173 Kessels AG, Thywissen T, Beets-Tan RG. Tumour ADC 174 measurements in rectal cancer: effect of ROI methods $\quad 175$ on ADC values and interobserver variability. Eur Radiol 176 2011;21:2567-74.

12. Iannicelli E, Di Pietropaolo M, Pilozzi E, Osti MF, 178 Valentino M, Masoni L, Ferri M. Value of diffusion- $\quad 179$ weighted MRI and apparent diffusion coefficient $\quad 180$ measurements for predicting the response of locally 181 advanced rectal cancer to neoadjuvant chemoradiotherapy. 182 Abdom Radiol (NY) 2016;41:1906-17. 183

13. Kaur H, Choi H, You YN, Rauch GM, Jensen CT, Hou 184 P, Chang GJ, Skibber JM, Ernst RD. MR imaging for 185 preoperative evaluation of primary rectal cancer: practical considerations. Radiographics 2012;32:389-409. 